\title{
Glycolysis and Lactate Dehydrogenase A in Iron-Mediated Suppression of Osteoblast Function
}

\author{
Pete Hunter, Daniel Edwards ${ }^{1}$, Christopher Miller ${ }^{1}$, Erica L. Clinkenbeard ${ }^{1}$ \\ ${ }^{1}$ Department of Medical and Molecular Genetics \\ Indiana University School of Medicine | Indianapolis, IN 46202
}

\begin{abstract}
Background/Objective:
An estimated 37 million Americans have Chronic Kidney Disease (CKD), a condition in characterized by gradual decline in kidney function ${ }^{1}$. Patients with CKD are often afflicted with skeletal fractures, increasing morbidity and mortality. CKD has also shown a strong correlation with iron-deficiency anemia. The underlying mechanisms of how iron-deficiency anemia of CKD affects bone loss are not well understood. Based on RNA-sequencing results, we hypothesize that lactate dehydrogenase $\mathrm{A}$ (LDHA) may play a role in iron-deficiency mediated suppression of osteoblast differentiation and function.
\end{abstract}

\section{Methods:}

Mouse Progenitor Cells (MPC2) were incubated in osteogenic media along with deferoxamine (DFO) to induce differentiation in chronic iron deficiency; samples were collected after 7 and 14 days. Quantitative real-time PCR and western blot were used to validate LDHA mRNA and protein levels in DFO treated MPCs versus control cells. RNA levels of osteoblast genes and LDHA were also assessed in a pre-clinical mouse model of CKD.

\section{Results:}

In vitro, MPCs cultured in DFO media showed a significant increase of LDHA mRNA at 7 days $(p=0.015)$ and returned to near control levels by day 14 . Western blots showed a slight increase of total LDHA protein in DFO treated MPCs at 7 days and a large increase of protein at the 14day mark $(p=0.051)$. In vivo, CKD bone marrow showed a reduction in osteoblast gene expression (osteocalcin and type 1 collagen; $p<0.05$ ). LDHA mRNA expression was increased in CKD mice bone marrow when compared to wild-type mice $(p=0.051)$, suggesting an inverse relationship.

\section{Conclusion and Potential Impact:}

Inappropriate activation of glycolysis and LDHA appears to play a role in iron-deficiency mediated suppression of osteoblast function in relation to CKD, both in vitro and in vivo. Further exploration of this relationship could be critical to the development of improved treatment options to maintain bone homeostasis during CKD. 